\section{RMD Open}

Rheumatic \&

Musculoskeletal Diseases
To cite: Micheroli $\mathrm{R}$, Elhai M, Edalat $\mathrm{S}$, et al. Role of synovial fibroblast subsets across synovial pathotypes in rheumatoid arthritis: a deconvolution analysis. RMD Open 2022;8:e001949. doi:10.1136/ rmdopen-2021-001949

- Additional supplemental material is published online only. To view, please visit the journal online (http://dx.doi.org/10. 1136/rmdopen-2021-001949).

Received 18 September 2021 Accepted 1 December 2021

Check for updates

(C) Author(s) (or their employer(s)) 2022. Re-use permitted under CC BY-NC. No commercial re-use. See rights and permissions. Published by BMJ.

For numbered affiliations see end of article.

Correspondence to Dr Raphael Micheroli; raphael.micheroli@usz.ch

\title{
Role of synovial fibroblast subsets across synovial pathotypes in rheumatoid arthritis: a deconvolution analysis
}

\section{ABSTRACT}

Objectives To integrate published single-cell RNA sequencing (scRNA-seq) data and assess the contribution of synovial fibroblast (SF) subsets to synovial pathotypes and respective clinical characteristics in treatment-naïve early arthritis. Methods In this in silico study, we integrated scRNA-seq data from published studies with additional unpublished in-house data. Standard Seurat, Harmony and Liger workflow was performed for integration and differential gene expression analysis. We estimated single cell type proportions in bulk RNA-seq data (deconvolution) from synovial tissue from 87 treatment-naïve early arthritis patients in the Pathobiology of Early Arthritis Cohort using MuSiC. SF proportions across synovial pathotypes (fibroid, lymphoid and myeloid) and relationship of disease activity measurements across different synovial pathotypes were assessed.

Results We identified four SF clusters with respective marker genes: PRG4 $4^{+}$SF (CD55, MMP3, PRG4, $T H Y 1^{\text {neg }}$ ); CXCL12 ${ }^{+}$SF (CXCL12, CCL2, ADAMTS1, THY1 ${ }^{\text {low }) ; ~ P O S T N ~}{ }^{+}$ SF (POSTN, collagen genes, THY1); CXCL14+ SF (CXCL14, C3, CD34, ASPN, THY1) that correspond to lining (PRG4 ${ }^{+}$ SF) and sublining (CXCL12 $2^{+}$SF, POSTN ${ }^{+}+$and $C X C L 14^{+}$ SF) SF subsets. CXCL12 $2^{+}$SF and POSTN ${ }^{+}+$were most prominent in the fibroid while $P R G 4^{+}$SF appeared highest in the myeloid pathotype. Corresponding, lining assessed by histology (assessed by Krenn-Score) was thicker in the myeloid, but also in the lymphoid pathotype + the fibroid pathotype. $P R G 4^{+}$SF correlated positively with disease severity parameters in the fibroid, POSTN ${ }^{+}$SF in the lymphoid pathotype whereas $\mathrm{CXCL} 14^{+} \mathrm{SF}$ showed negative association with disease severity in all pathotypes.

Conclusion This study shows a so far unexplored association between distinct synovial pathologies and SF subtypes defined by scRNA-seq. The knowledge of the diverse interplay of SF with immune cells will advance opportunities for tailored targeted treatments.

\section{INTRODUCTION}

During the development of rheumatoid arthritis (RA), synovial architecture and cellular content change dramatically. The thin

\section{Key messages}

What is already known about this subject?

- Different studies were able to show the presence of diverse synovial fibroblast (SF) subsets in the rheumatoid arthritis (RA) synovium using single-cell RNA sequencing.

- In RA, three distinct synovial pathotypes based on the presence of immune cells have been identified which could be connected with specific clinical parameters and outcome measures.

What does this study add?

- We could confirm the presence of specific SF subsets in the synovium and showed the feasibility of integration of single-cell RNA sequencing data from different resources.

- By deconvolution of bulk RNAseq, we show differences in the proportion of SF subtypes within different pathotypes and specific correlations of the various SF subtypes with disease activity/severity dependent on the histological pathotype.

How might this impact on clinical practice or further developments?

- This so far unexplored connection between the histological pathotype and SF subtypes guides further research to understand the impact of the presence of immune cells on SF subtypes, and thus opens new avenues for targeted treatment according to the synovial composition.

membrane lining the joint synovium becomes an inflamed, hyperplastic and invasive tissue mass of infiltrating cells-most prominent synovial fibroblasts (SF) - that ultimately lead to joint destruction. ${ }^{1}$ In the sublining, the presence of infiltrating immune cells, expanded SF and increased vascularity are characteristic for RA synovium.

Previous research revealed the presence of three distinct synovial pathotypes based on 
cellular and molecular analysis of synovial tissue: ${ }^{2}$ (1) lymphomyeloid dominated by the presence of B-cells in addition to myeloid cells (hereafter lymphoid); (2) diffuse-myeloid with myeloid lineage predominance but poor in B cells (hereafter myeloid) and (3) pauciimmune characterised by scanty immune cells and prevalent stromal cells (hereafter fibroid). Correlation analysis demonstrated that elevation of myeloid-associated and lymphoid-associated gene expression strongly correlated with disease activity, acute phase reactants and response to disease-modifying antirheumatic drugs at six months. ${ }^{3}$ Patients with predominant fibroid pathology showed less severe disease activity and radiographic progression, but poor treatment response. ${ }^{3}$

New single cell sequencing tools, including singlecell RNA sequencing (scRNA-seq), provide deep insight into tissue biology at the cell-state level, uncovering a diversity of synovial lymphoid, myeloid and stromal cell populations. ${ }^{4-7}$

Despite this expanded knowledge and the fact that already early studies showed the presence of different synovitis subtypes ${ }^{8}$ and the potential of SF to adapt to the inflammatory status in the synovium, ${ }^{9}$ the relationship between SF subsets and the diversity of tissue pathology that is, different synovial pathotypes is unknown. Therefore, we first aimed to confirm specific subsets of SF in synovial tissue by integration of published and unpublished scRNA-seq data. We then assessed the contribution of the defined SF subsets to synovial pathotypes and clinical characteristics using deconvolution analysis of bulk RNA-seq in treatment-naïve early arthritis synovial tissue.

\section{MATERIAL/METHODS}

\section{ScRNA-seq of synovial biopsies}

The used publicly available datasets are described in online supplemental text 1 . Following, we report the additionally used in-house datasets. Synovial biopsies were obtained from one wrist and one metacarpophalangeal (MCP) joint of patients with active RA by ultrasound guided fine-needle biopsy after obtaining informed consent. Both female patients fulfilled the ACR/EULAR classification criteria ${ }^{10}$ for RA and had no concomitant therapy during the time of the biopsy. Synovial biopsies were washed with phosphate-buffered saline, mechanically minced and enzymatically digested using Liberase TL $(100 \mu \mathrm{g} / \mathrm{mL}$; Roche) and DNAse I $(100 \mu \mathrm{g} / \mathrm{mL}$; Roche) in RPMI 1640 cell culture medium (Thermo Fisher) for $30 \mathrm{~min}$ at $37^{\circ} \mathrm{C}$. After stopping the digestion process with fetal calf serum, erythrocytes were lysed with Red Blood Cell Lysis solution (Milteny Biotec). Cells were washed and counted on a LUNA automated cell counter (Logos Biosystems). A total of 15000 unsorted synovial cells per patient were prepared for single cell analysis using the Chromium Single Cell 3' GEM, Library \& Gel Bead Kit v3, the Chromium Chip B Single Cell Kit (10× Genomics) and the Chromium controller (all 10× Genomic). Libraries were sequenced on the
Illumina NovaSeq instrument to a sequence depth of 20 000-70 000 reads per cell. CellRanger (V.2.0.2) from $10 \times$ Genomics was used to demultiplex, align the reads to Ensembl reference build GRCh38.p13 and collapse unique molecular identifiers.

\section{Integration of scRNA-seq datasets}

Standard Seurat (V.3.3) for R (V.3.6) protocol-a very well established package to analyse scRNA-seq data-was used for the integration. ${ }^{11}$ In a sensitivity analysis, the integration step was additionally performed with Liger (V.1.0) ${ }^{12}$ as well as Harmony (V.0.1) ${ }^{13}$ since these three methods have been found to be superior compared with other integration protocols. ${ }^{14}$ If not otherwise stated, default settings were used for each integration method. Online supplementary text 1 gives further information about the integration process.

To identify differentially expressed genes between groups of cells, we used the Wilcoxon rank-sum test. We used a minimum $\log 2 \mathrm{FC}$ of 0.25 for average expression of genes in a cluster relative to the average expression in all other clusters combined. To take multiple testing into account, $p$ values were adjusted by false discovery rate (FDR) controlling. Significant Marker genes (adjusted $\mathrm{p}<0.05$ ) were sorted by average log2 FC. KEGG Pathways of the Marker Genes were estimated with clusterProfiler $\mathrm{R}$ package.

\section{Pseudotime trajectory analysis}

Monocle $2 \mathrm{R}$ package (V.2.2) was used to perform pseudotime trajectory analysis. ${ }^{15}$ Monocle applies advanced machine learning methods to find transcriptomic changes each cell goes through as part of a dynamic biological process. Once the overall 'trajectory' of gene expression changes has been identified, each cell gets placed at its proper position in the trajectory. Furthermore, Monocle tracks changes as a function of progress along the trajectory, which is termed "pseudotime". After ordering of cells along the trajectory, genes that change as a function of pseudotime will be identified. Most significant genes with similar trends over pseudotime were grouped together with the function 'plot_pseudotime_heatmap'. All tasks were performed with default settings. ${ }^{16}$

\section{Deconvolution analysis of SF subsets across synovial pathotypes}

Bulk RNA-seq data from synovial tissue as well as corresponding cellular (immunohistology with pathotype grading) and clinical characteristics were available from 87 treatment-naive early RA patients $(<12$ months symptoms duration) in the Pathobiology of Early Arthritis Cohort (PEAC).$^{17}$ Information about the PEAC-Cohort was previously described. ${ }^{23}$ From the 87 patients, 16 had a fibroid, 45 a lymphoid and 20 a myeloid pathotype; in 6 patients the grading was not possible.

Fastq files were downloaded fromthe European Bioinformatics Institute with the accession code E-MTAB-6141 and were mapped to hg19 and sequence reads assigned 


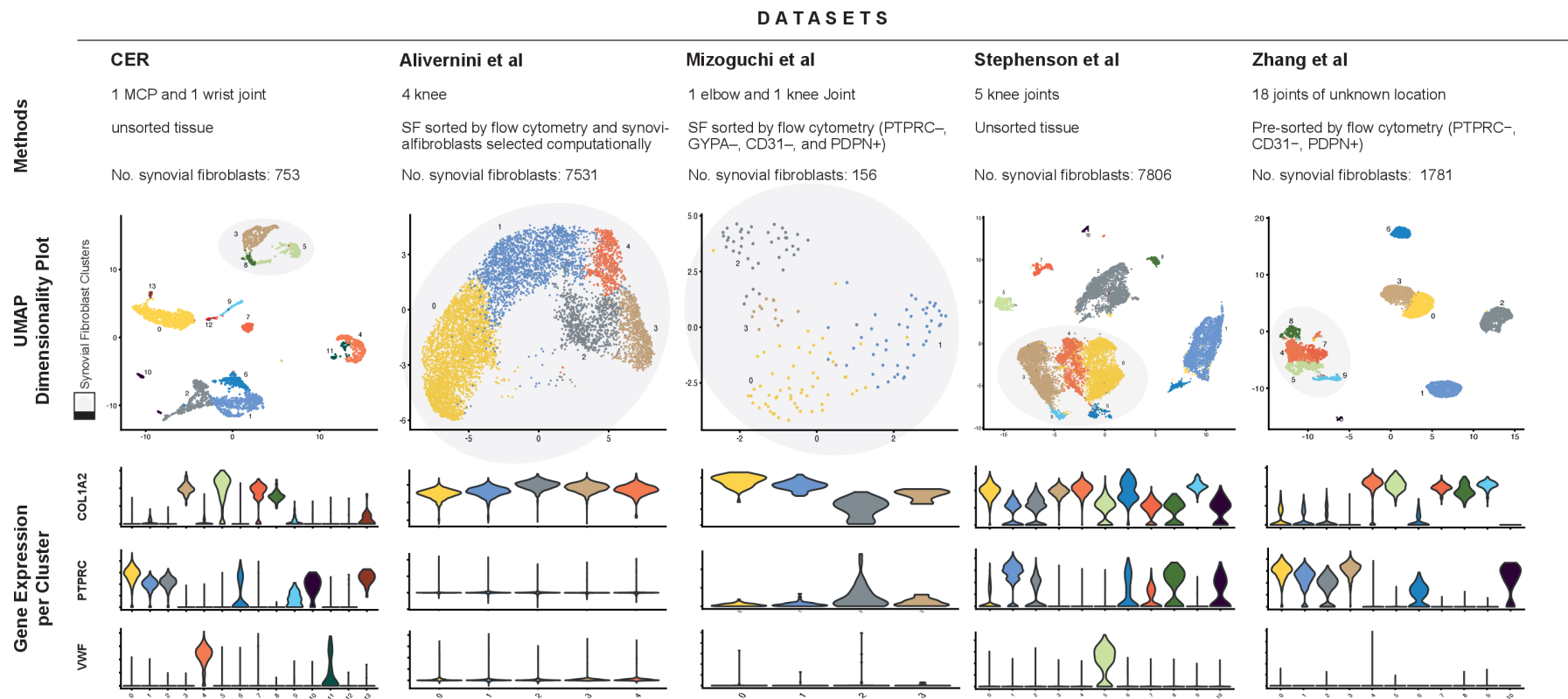

Figure 1 Individual synovial tissue datasets with respective used methods and number of synovial fibroblasts (SF), UMAP visualisation and SF positive (COL1A2) and negative (PTPRC, vWF) marker genes. Selected SF clusters in unsorted datasets are marked in grey in the UMAP plot. CER $=$ Center of Experimental Rheumatology Zurich, UMAP = Uniform Manifold Approximation and Projection for Dimension Reduction. UMAP; Uniform Manifold Approximation and Projection for Dimension Reduction.

to genomic features using $\operatorname{STAR}^{18}$ and featureCounts, ${ }^{19}$ respectively.

MuSiC R package was used to perform the deconvolution analysis. ${ }^{20} \mathrm{MuSiC}$ uses cell-type specific gene expression from scRNA-seq data to characterise cell type compositions in bulk RNA-seq. A standard protocol was used to estimate cell type proportions in bulk tissue. ${ }^{21}$

Pairwise-Wilcox test was used to calculate pairwise comparisons between group levels. SF cluster proportions were analysed for correlation with disease activity measures across different synovial pathotypes.

$\mathrm{P}$ values were adjusted for multiple testing by FDR controlling.

\section{Histological analysis}

The pathotype and Krenn lining score ${ }^{22}$ were assessed in a subset of 69 synovial samples from patients with RA as previously described. ${ }^{2}$

\section{RESULTS}

\section{Integration of five scRNA-seq datasets}

We first collected scRNA-seq data from 31 RA synovial tissues of four published studies and of an in-house dataset (figure 1 and online supplementary text 1 ). SFs were selected based on the presence of COL1A2 and absence of PTPRC (CD45, leucocyte marker) and VWF (von Willebrand factor, endothelial cell marker) gene expression. Uniform Manifold Approximation and Projection for Dimension Reduction (UMAP) visualisation showed that the expression of these genes was uniformly present in neighbouring clusters apart from cluster 7 in the samples from the Center of Experimental
Rheumatology Zurich (CER). The main marker genes of cluster 7 were suggestive of a smooth muscle origin (eg, MYH11, ACTA1 and ACTA2) and thus the cluster was excluded from further analysis. The studies of Alivernini et $a l^{7}$ and Stephenson et $a l^{4}$ had a substantially higher number of SF cells compared with the other datasets (figure 1). To correct for this imbalance, we randomly reduced the number of identified $\mathrm{SF}$ of these studies to 3000 and used the remaining $8693 \mathrm{SF}$ for further analysis.

\section{Generation SF signatures}

Integration and clustering of the identified SF cells using Seurat, Liger or Harmony identified similar clusters of $\mathrm{SF}$ illustrated by the expression of marker genes determined in previous studies: CD55, THY1, CD34, POSTN and HLA-DRA (figure 2A). In all three approaches, four SF subtypes were distinguishable (figure 2A). The top 20 marker genes of the Seurat, Liger and Harmony determined SF clusters are presented in online supplemental tables 1-3.

The first subtype ( $\left.P R G 4^{+} \mathrm{SF}\right)$ expressed high levels of CD55, MMP3, PRG4 and FN1, and was THY1 negative. This subtype was previously suggested to reside in the lining layer of the synovium. ${ }^{4-7}$ The second subtype $\left(C X C L 12^{+} \mathrm{SF}\right)$ expressed CXCL12, CCL2 and ADAMTS1 and had the lowest THY1 expression of the three THY1 positive subtypes. The third subtype $\left(P O S T N^{+} \mathrm{SF}\right)$ showed high expression of POSTN and collagen genes. This subtype expressed intermediate levels of THY1. The fourth subtype (CXCL14 $4^{+} \mathrm{SF}$ ) expressed high levels of CXCL14, C3, ASPN, THY1 and CD34. Liger identified an additional fifth subtype. This subtype contained CD55 
UMAP Dimension Plots

A

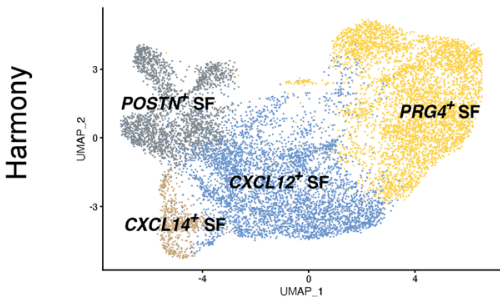

- Alivernini $\bullet$ Mizoguchi $\bullet$ Stephenson
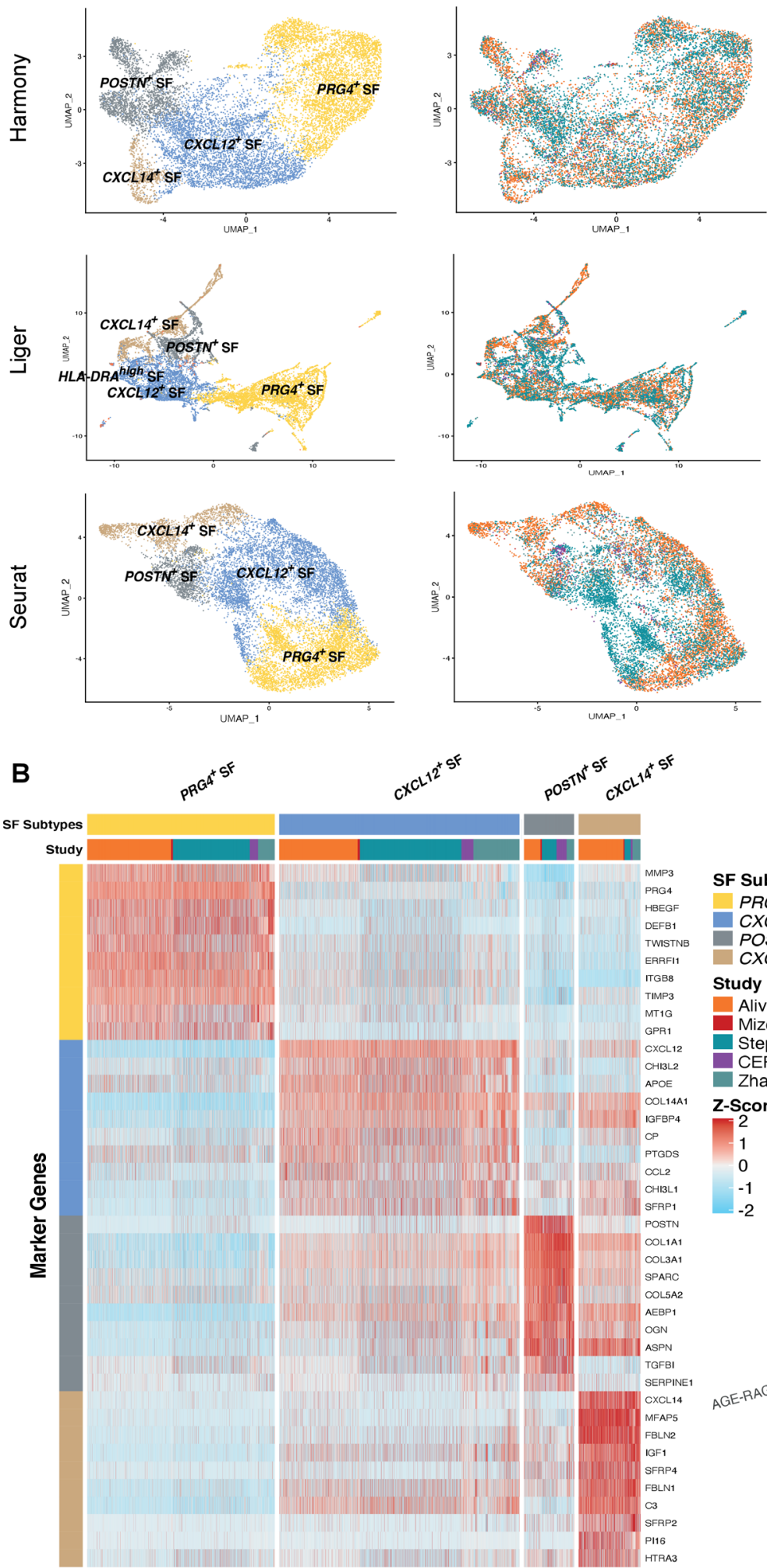

-1
-2

Gene Expression per Cluster
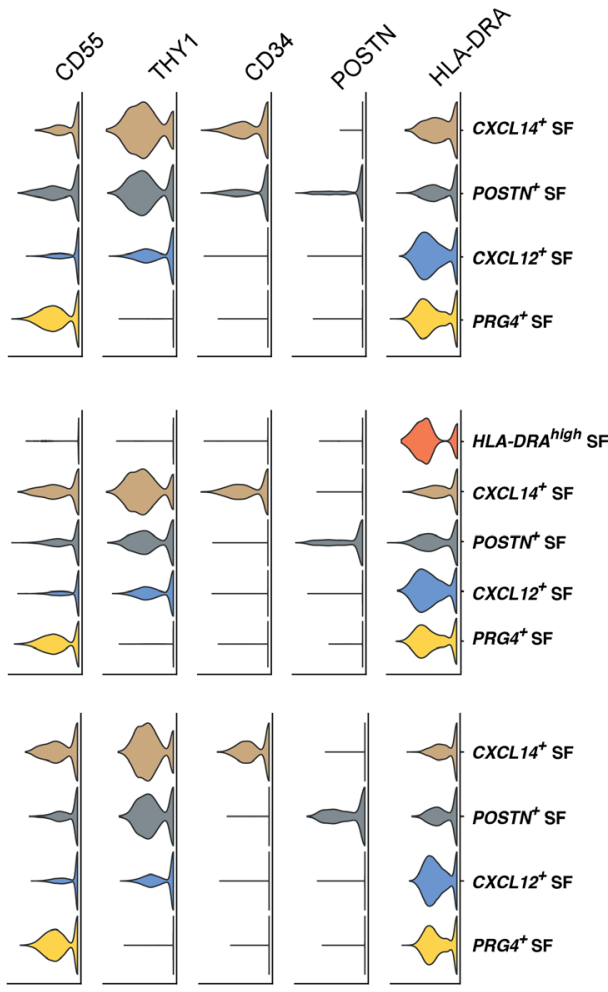

Cluster

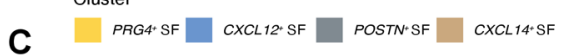

c

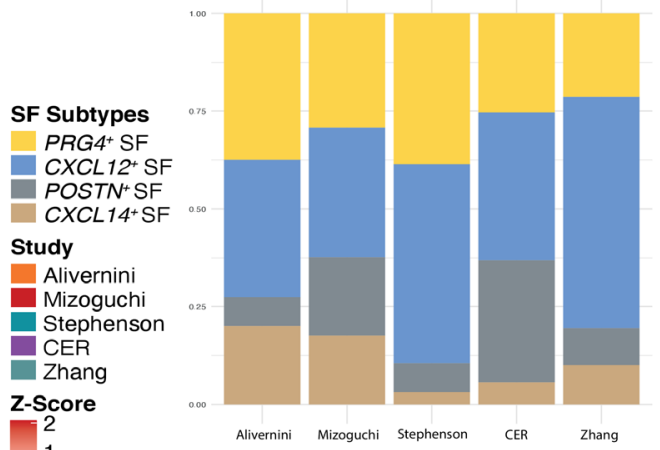

\section{D}

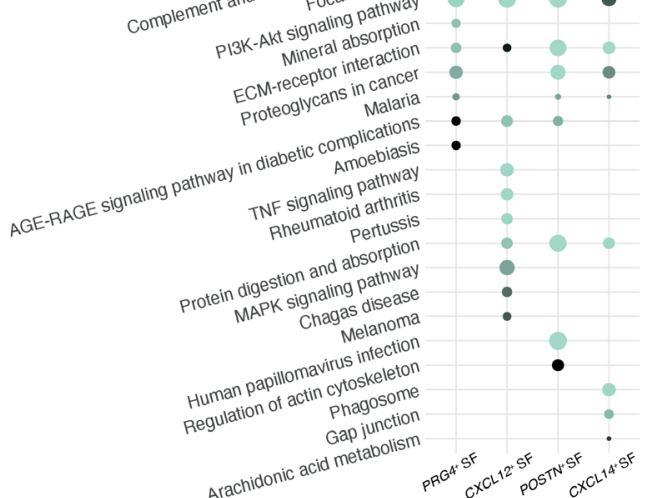

Figure 2 Identification of synovial fibroblast (SF) subsets (A) different integration methods with UMAP visualisation, grouping of cells according to datasets and violinplots showing the gene expression per cluster of CD55, THY1, CD34, POSTN and HLA$D R A$. (B) Heatmap of the 10 most significant marker genes of each SF cluster (via Seurat). (C) Distribution of seurat clusters across the different datasets. (D) KEGG pathways enrichment analysis across SF clusters. Top 20 pathways are shown. UMAP; Uniform Manifold Approximation and Projection for Dimension Reduction. 
and THY1 negative cells with high expression of HLA$D R A$ (figure 2A). The cluster represented a minor population of cells, some of which were also scattered across the other four subtypes. Therefore, this subtype was not considered in the further analysis.

UMAP with colour-coding according to the data source confirmed that the four subtypes were derived from data across all data sets (figure 2A). However, the distribution of the SF subsets differed between the studies (figure 2C). The variation in distribution of the subtypes may be due to the differences in material and methods used. But even in the two studies with the most similar approach (Mizoguchi et a $a \tilde{l}$ and Zhang et $a l^{6}$ ) the distribution of the subtypes varied and two studies with a very different approach (CER and Mizoguchi et $a \bar{\varphi}$ ) showed similar patterns of SF subtype distribution. Differences in patient selection (disease stage and activity), previous and current treatment and joint location might contribute to variation in subtype distribution between the studies.

We then performed KEGG pathway analysis across the SF subtypes (figure 2D, online supplementary table 4). $P R G 4^{+}$SF genes were enriched for terms such as +'focal adhesion', 'ECM-receptor interaction' and 'mineral absorption', which supported the assignment of these $\mathrm{SF}$ to the synovial lining layer. CXCL $12^{+} \mathrm{SF}$ genes were enriched for different pathways associated with proinflammatory states (+ 'RA', 'TNF signalling pathway', 'MAPK signalling pathway'). Pathways that were unique for $P_{O S T N}^{+}$+ were 'human papillomavirus infection' (included genes: COL1A1, COL3A1, COL1A2, ACTN1, $L A M B 1$ ) and 'regulation of actin cytoskeleton' (included genes: ITGA10, MYLK, ACTN1, MYH10, PDGFRB, MYL9, ENAH, ITGA11, ITGB5). Most significant pathways of $\mathrm{CXCL14^{+ }}$ + were 'phagosome', 'gap-junction' and 'arachidonic acid metabolism'.

\section{Assessment of dynamic relationships between SF subtypes}

After the investigation of different gene expression profiles of the SF clusters, we assessed the relationships between the different clusters using monocle 2 (figure 3). A continuum over five different cell states was found by advanced machine learning technique (figure $3 \mathrm{~A}$ ). When overlaid with the SF subtypes, it appeared that the cells dispersed along the trajectory beginning in $P R G 4^{+}+$over $C X C L 12^{+}+$to $C X C L 14^{+} \mathrm{SF}$ (figure 3B and C). CXCL12 $2^{+} \mathrm{SF}$ additionally formed two small branches of cell states. The state at one side of the trajectory - with mostly containing $\mathrm{PRG4}^{+} \mathrm{SF}$ - was defined as starting point for further analysis. Differential expression analysis over pseudotime identified several genes that changed significantly during the transition (online supplementary table 5). Figure 3D shows the 50 most significant changing genes over the pseudotime trajectory in a heatmap. Included in these genes are THY1 and PRGA, genes that were previously described as relevant positional markers of SF in the synovium ${ }^{23}$ in sublining and lining SF, respectively (figure 3D and E).
In summary, we successfully integrated five datasets with which we could recapitulate previously identified SF subtypes as well as the plasticity of these cellular states according to their location within the synovium.

\section{Distribution of SF phenotypes across synovial pathotypes}

We then integrated and clustered all synovial cells to be able to perform the deconvolution analysis of bulk transcriptomics data of synovial tissues (online supplementary table 6 and supplementary figure 1). Deconvolution analysis revealed a distribution of the different cell types as the respective pathotypes would suggest (figure 4A). The proportion of SF was highest in the fibroid pathotype, while the lymphoid pathotype showed a clear enrichment in myeloid cells, plasma cells, B cells and T cells (figure 4A). The myeloid pathotype was denoted by high percentage of myeloid cells but absence of $\mathrm{T}$ and $\mathrm{B}$ cells. Overall, these results confirmed the accuracy of our approach.

Analysis of SF subtype enrichment within the various pathotypes suggested that POSTN ${ }^{+}+$and CXCL14 $4^{+}+$ are most prominent in the fibroid pathotype, while the proportion of $\mathrm{PRGA}^{+} \mathrm{SF}$ expanded in the myeloid pathotype (+). Statistical analysis confirmed that $P R G 4^{+}+$had higher proportions in the myeloid pathotype compared with the fibroid pathotype (figure 4B). Accordingly, lining thickness assessed by histology was more pronounced in the myeloid, but also in the lymphoid pathotype compared with the fibroid pathotype (online supplementary figure 2). CXCL12 $2^{+}+$and POSTN ${ }^{+}+$had the highest proportions in the fibroid pathotype, followed by the lymphoid pathotype in $C X C L 12^{+}+$and the myeloid pathotype in $\operatorname{POSTN}^{+} \mathrm{SF}$, respectively (+).

In summary, these data suggest an expansion of lining $P R G 4^{+}$SF fibroblasts in the myeloid pathotype and increased proportions of CXCL12 $2^{+} \mathrm{SF}$ and POSTN $^{+}+$in the fibroid pathotype.

\section{Correlation of SF subtypes with clinical characteristics}

To assess the connection of the different subtypes with pathological processes and clinical symptoms in RA, we correlated demographic and clinical data with the presence of the SF subtypes (figure 4C,D, online supplementary table 7). Proportions of SF were neither different between men and women nor between seropositive and seronegative patients or different age groups. Without differentiating the patients according to the synovial pathotype, there was a significant negative relationship between CXCL $14^{+}+$and swollen joint count $(\mathrm{R}=-0.61$, adjusted $p=$ value 0.047 ) as well as Disease Activity Score-28 (DAS28 $(\mathrm{R}=-0.64$, adjusted $\mathrm{p}<0.001)$. Also, within the different pathotypes, proportions of $C X C L 14^{+}+$were generally negatively correlated with clinical parameters. Specifically in the myeloid pathotype, CXCL14+ SF showed the strongest negative association with DAS28 $(\mathrm{R}=-0.98 ; \mathrm{p}=0.0025$, adjusted $\mathrm{p}=0.047)$.

Even though $C X C L 12^{+}+$was characterised by inflammatory signalling pathways, its presence did not correlate 
A

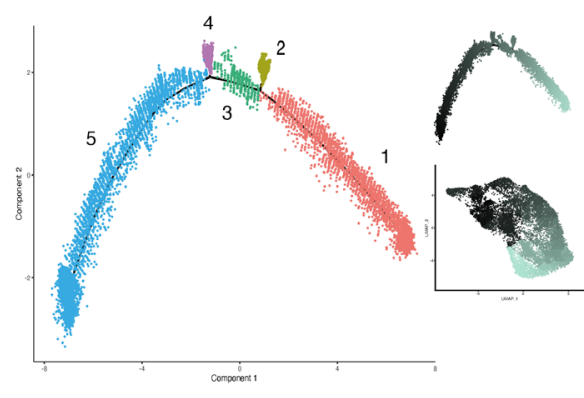

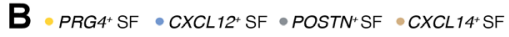

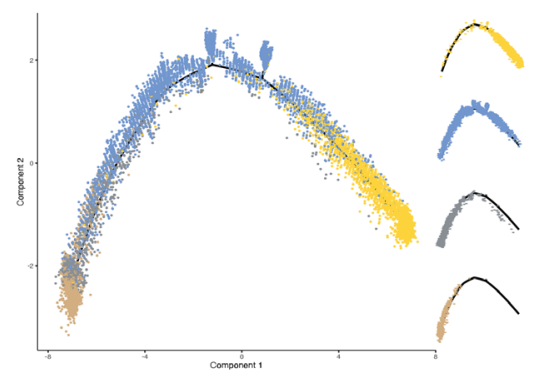

C
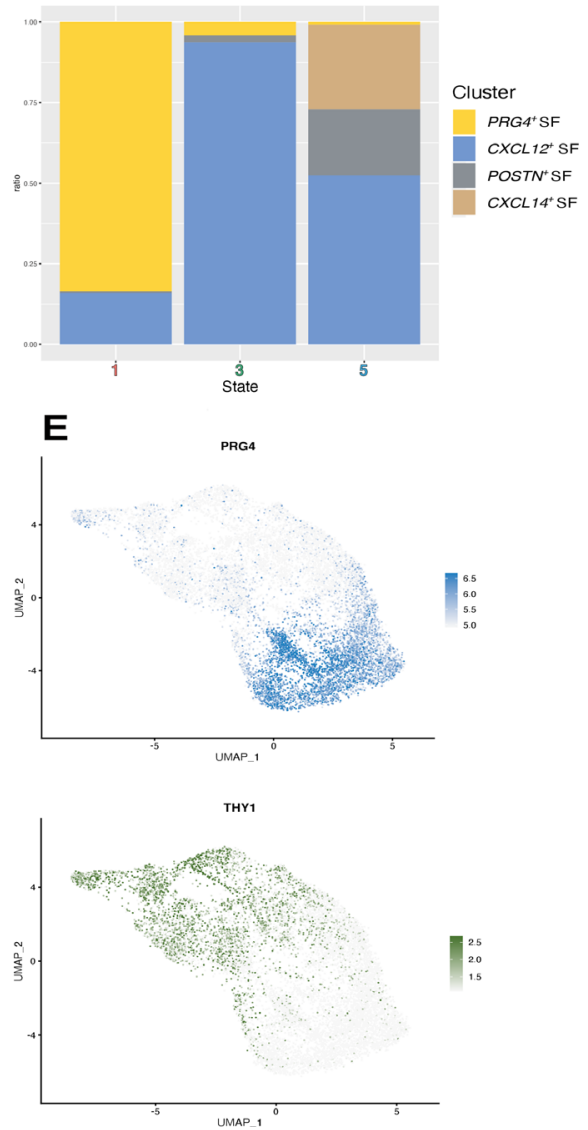

D
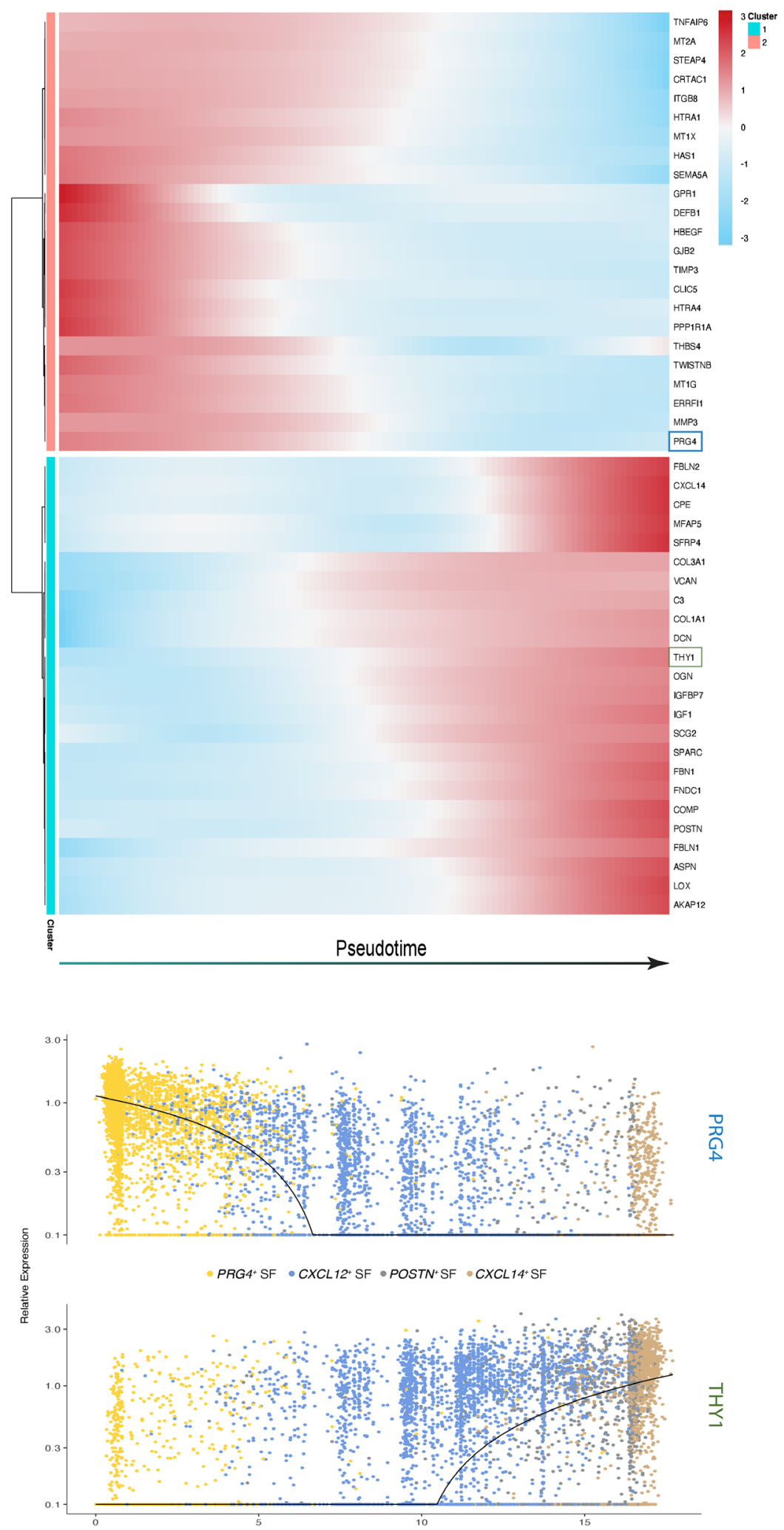

Figure 3 Pseudotime analysis of synovial fibroblast (SF) subsets. (A) Visualisation of the trajectory states of all SF. Top and middle right shows the pseudotime trajectory in the reduced dimension and UMAP visualisation. (B) Distribution of the SF cluster along the trajectory (right split by cluster). (C) Distribution of SF clusters in the main trajectory states 1, 3 and 5. (D) Most significant genes that covary across pseudotime split in two clusters (state one left, state five right). (E) Plot of gene expression levels of PRG4 and THY1 in the UMAP of the SF clusters on the left and along the pseudotime trajectory on the right. UMAP = Uniform Manifold Approximation and Projection for Dimension Reduction. 


\section{Figure 4}

\section{A}

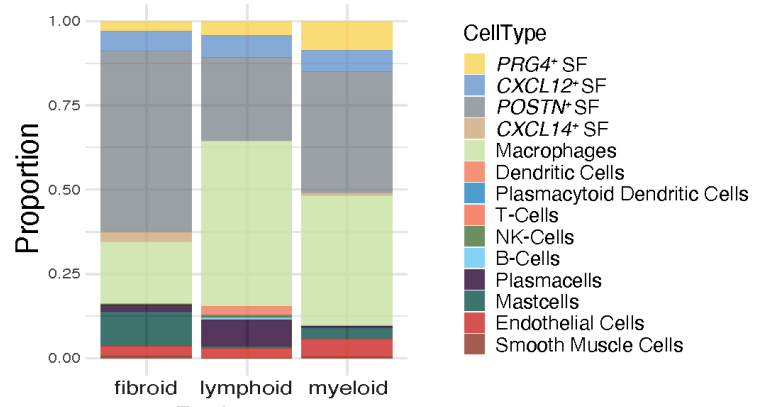

Pathotype

B FDR adjusted p-values for wilcoxon rank sum tests are shown

Pathotype

CXCL12+SF

$\bigoplus_{\text {fibroid }}$ lymphoid myeloid
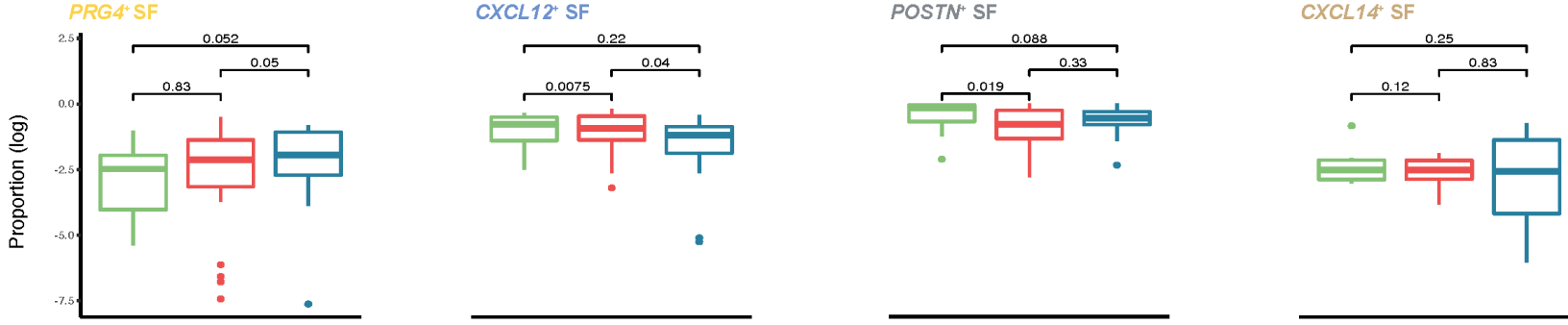

C correlation (R) with FDR adjusted p-values are shown

Pathotype PRG 4 SF CXCL12+ SF

- tibroid $=$ lymphoid $=$ myeloid
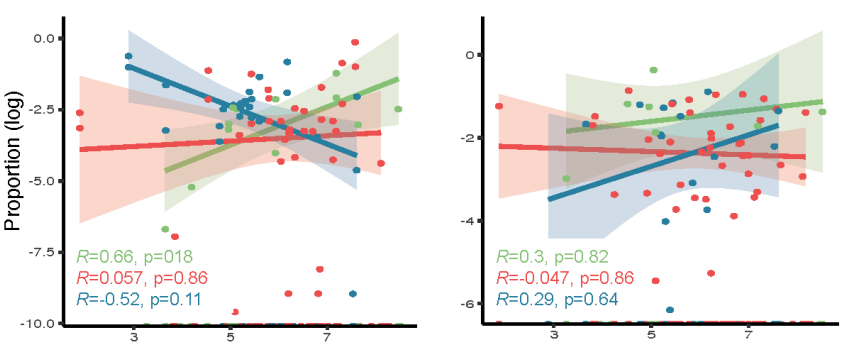

POSTN $N^{+}$SF

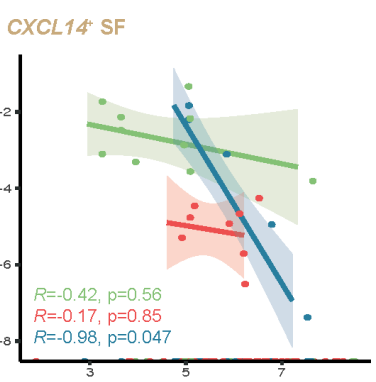

D

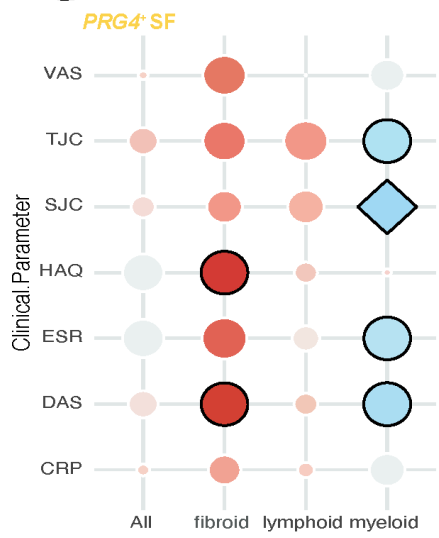

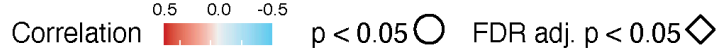

CXCL12+ SF

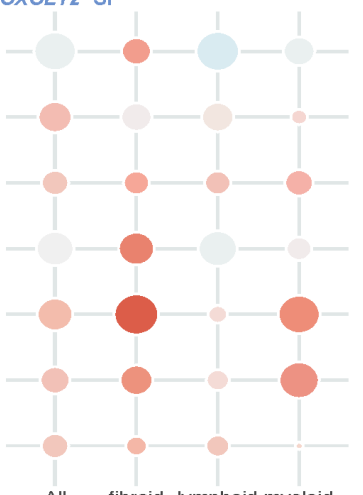

POSTN $N^{+}$SF

Al
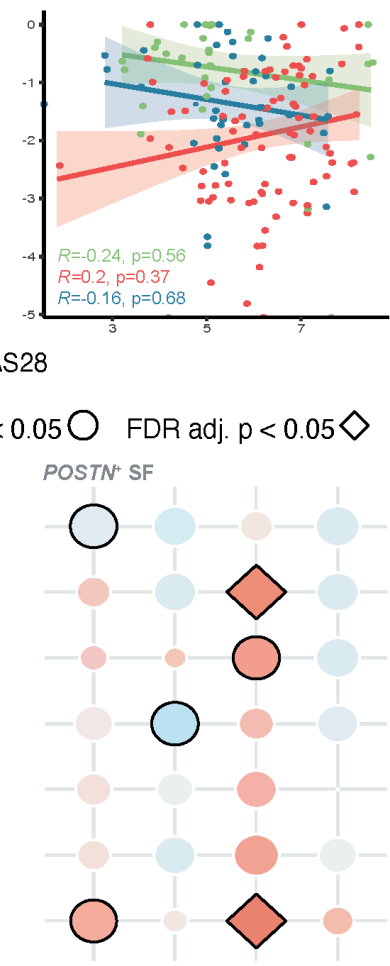

AS28

Pathotype

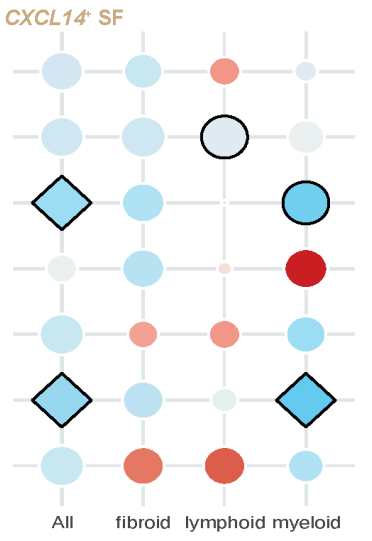

Figure 4 Synovial fibroblasts (SF) across synovial pathotypes and comparison with disease activity measurements. (A) Distribution of different cell types between the different synovial pathotypes. (B) Proportion of the different SF subtypes within the pathotypes. (C) Correlation between disease activity measurements and SF proportions across synovial pathotypes. (D) Dotplot with Pearson correlation of different clinical parameters with SF subtypes across pathotypes. DAS; disease activity score. ESR; erythrocyte sedimentation rate. FDR; false discovery rate. HAQ; health assessment questionnaire. SJC; swollen joint count. TJC; tender joint count. VAS; visual analog scale. 
with any clinical symptoms. Strikingly for $P R G 4^{+}+$and $\operatorname{POSTN}^{+} \mathrm{SF}$, correlations were opposite in the different pathotypes. In the lymphoid pathotype, increasing $P_{O S T N}{ }^{S}$ SF proportions were related with higher tender and swollen joint counts and CRP levels, but in the fibroid and lymphoid pathotype these correlations tended to be reversed (figure $4 \mathrm{D}$ and online supplementary table 7 ). Similarly for $P R G 4^{+} \mathrm{SF}$, in the fibroid pathotype a positive relationship with disease activity $(+=0.66, \mathrm{p}=0.02$, adjusted $\mathrm{p}=0.18$ ) and with the Health Assessment Questionnaire $(\mathrm{R}=0.67, \mathrm{p}=0.017$, adjusted $\mathrm{p}=0.18)$ was seen, but in the myeloid pathotype a negative correlation with disease activity $(\mathrm{R}=-0.52 ; \mathrm{p}=0.007$, adjusted $\mathrm{p}=0.11)$, particularly a negative association with the swollen tender joint count $(\mathrm{R}=-0.63 ; \mathrm{p}<0.001$; adjusted $\mathrm{p}=0.023)$ was measured (figure 4D). Together these data point to a substantial influence of immune cells on shaping gene expression of $P R G 4^{+}+$and $P O S T N^{+}+$in arthritic synovium.

We also looked at the relationship of other synovial cell types with DAS28 (online supplementary figure 3); however, without further division of these additional cell types in subtypes, no significant relationship was found.

\section{DISCUSSION}

In this study, we were able to integrate synovial single-cell data from different sources and protocols and confirmed the presence of four distinct SF subpopulations in RA synovium. ${ }^{4-7}$ Furthermore, we could show that the different SF subtypes vary in their distribution within pathotypes and correlate with distinct clinical disease characteristics dependent on the pathotype. Thus, with our data, we revealed a presently unexplored connection between the SF subtypes and the type of immune cell infiltration in RA synovium.

The SF subtypes that we defined with the integrated dataset are largely in line with previously identified SF subtypes. All the previously published datasets obtained a subset of SF with high expression of CD55 and absence of THY1 expression. Further analysis-using staining of CD55 in the synovium ${ }^{4}$ and high expression of known SF lining genes ${ }^{5}$ - concluded that these cells most likely represent lining SF. In the fibroid pathotype, the proportions of $\mathrm{PRG}^{+} \mathrm{SF}$ showed the most positive correlations with clinical parameters, leading to the hypothesis that in low presence of immune cells the lining $P R G 4^{+} \mathrm{SF}$ subset might play a stronger role in driving RA symptoms as compared with the myeloid pathotype, where proportions of PRG4+ SF mostly negatively correlated with clinical parameters. It has been postulated that lining SF mediate tissue damage and sublining SF coordinate inflammatory responses. ${ }^{24}$ Serum $M M P 3$ - the most significant marker gene of $P R G 4^{+} \mathrm{SF}$ - has a known association with disease activity and joint damage. ${ }^{2526}$ However, previous studies from the PEAC cohort found that the fibroid pathotype was associated with less radiographic progression compared with the other pathotypes, ${ }^{2}$ implicating a role of additional cell types in mediating tissue destruction. Notably, $P R G 4^{+}$SF proportions were higher in the myeloid pathotype + the others. This is in concordance with a previous study showing that lining SFs are positively correlated with macrophage density. ${ }^{27}$ Surprisingly, $P R G 4^{+}$SF proportions negatively correlated with clinical parameters in the myeloid pathotype suggesting that the increased presence of macrophages might have a regulatory effect on this SF subtype.

CXCL $12^{+} \mathrm{SF}$ subset of integrated data set showed high expression of CXCL12 and HLA-DRA and no expression of $C D 34$, consistent with an SF subtype described by Alivernini $^{7}$ and Zhang ${ }^{6}+$, in which the corresponding associated genes were more highly expressed in leucocyte-rich RA than in $\mathrm{OA}^{6}$

The POSTN ${ }^{+} \mathrm{SF}$ subset was + by high expression of POSTN. Also, Mizoguchi et a $\tilde{\varphi}$ found high expression of POSTN in the CD34/THY subset and an association of the respective SF subset with proportion of infiltrated leucocytes, histological synovitis, and synovial hypertrophy by ultrasound. In accordance, we found a positive correlation of this SF subtype with clinical parameters of active RA, but only in the lymphoid pathotype suggesting that interaction with lymphocytes may influence the pathogenic role of this SF subtype.

The $C X C L 14^{+} \mathrm{SF}$ subset expressed $C D 34$, as well as CXCL14. Based on the expression of inflammatory cytokines and higher number of recruited peripheral blood monocytes in a transwell + assay, Mizoguchi et $a \check{e}$ suggested that $C D 34^{\text {pos }} \mathrm{SF}$ are mostly responsible for monocyte recruitment in inflamed synovial tissue. However, the latter analysis was done with cultured SF subsets, which might alter their behaviour. Alivernini $e t a l^{7}$ separated a $C D 34^{+}+$a $C X C L 14^{+} \mathrm{SF}$ subsets. The CXCL14 $\mathrm{SF}$ in Alivernini $e t a l^{7}$ was further characterised by high expression of GAS6, regulating the function of synovial tissue macrophages in remission $\left(\mathrm{MerTK}^{\mathrm{pos}} \mathrm{CD} 206^{\mathrm{pos}}\right.$ ). GAS6 expression in $\mathrm{CXCL14^{+ }}$ + was higher in remission compared with active RA in Alivernini et $a l^{7}{ }^{7}$ which points to a possible anti-inflammatory role of this SF subtype. In accordance, in our analysis the presence of the CXCL $14^{+}$ SF subtype consistently negatively correlated with clinical symptoms of RA. Thus, CXCL $14^{+} \mathrm{SF}$ might represent a regulatory SF subtype attenuating inflammation. Besides this assumed influence of immune cells on SF, the other way around is possible as well; SF could be the initial driver and attract immune cells via different mechanism. via different mechanisms.

Using a deconvolution method, we were able to estimate proportions of various cell types within specific synovial pathotypes in the PEAC cohort. Most interestingly, the fibroid pathotype was characterised not only by a majority of SF but also by a high proportion of mast cells, which has not been studied in this context so far.

The limitation of this study includes that deconvolution is only a computational model, and further single cell analysis within the pathotypes will have to be performed to validate our data. Furthermore, functional analyses of SF subtypes are needed to investigate the relation 
between different SF subsets within pathotypes in detail. Nonetheless, our study has brought important insights for further research in this direction.

In conclusion, our study shows a so far unexplored association between the type of immune cell infiltration and the formation of SF subtypes. Knowledge of the influence of different SF subtypes on joint inflammation will enhance our understanding of the pathogenesis of RA, open new avenues for tailored targeted treatment according to the pathotype and reveal therapeutic targets for influencing the activated stroma in RA.

\section{Author affiliations \\ ${ }^{1}$ Center of Experimental Rheumatology, Department of Rheumatology, University Hospital Zurich, Zurich, Switzerland \\ ${ }^{2}$ Research Into Inflammatory Arthritis Centre Versus Arthritis (RACE), Institute of Infection, Immunity and Inflammation, University of Glasgow, Glasgow, UK ${ }^{3}$ Centre for Experimental Medicine and Rheumatology, Barts and The London School of Medicine and Dentistry, William Harvey Research Institute, London, UK ${ }^{4}$ Department of Rheumatology, University Medical Center Ljubljana, Ljubljana, Slovenia}

Twitter Raphael Micheroli @ramicheroli and Caroline 0spelt @Caroline0spelt

Acknowledgements RM thanks the swiss rheumatology society and the medAlumni university Zurich.

Contributors RM, CO and CP conceived and designed the study; CO, ME, SE, MF-B and TK performed experiments; RM and KB took biopsies from the Zurich samples; RM was responsible for data analysis; MKS and LM provided the single cell dataset from glasgow; $\mathrm{RM}$ and $\mathrm{CO}$ analysed results; $\mathrm{RM}$ and $\mathrm{CO}$ wrote the manuscript; all authors participated in discussions and interpreting the results and approved the final draft for publication. RM and $\mathrm{CO}$ are the guarantors.

Funding The authors have not declared a specific grant for this research from any funding agency in the public, commercial or not-for-profit sectors.

Competing interests None declared.

Patient consent for publication Not applicable.

Ethics approval This study as approved by National Research Ethics Service Committee London- Dulwich, Reference number 05/Q0703/198.

Provenance and peer review Not commissioned; externally peer reviewed.

Data availability statement Data are available in a public, open access repository. All data relevant to the study are included in the article or uploaded as online supplemental information. All data relevant to the study are included in the article, uploaded as online supplemental information or already publicly available.

Open access This is an open access article distributed in accordance with the Creative Commons Attribution Non Commercial (CC BY-NC 4.0) license, which permits others to distribute, remix, adapt, build upon this work non-commercially, and license their derivative works on different terms, provided the original work is properly cited, appropriate credit is given, any changes made indicated, and the use is non-commercial. See: http://creativecommons.org/licenses/by-nc/4.0/.

\section{ORCID iDs}

Raphael Micheroli http://orcid.org/0000-0002-8918-7304

Adrian Ciurea http://orcid.org/0000-0002-7870-7132

Caroline 0spelt http://orcid.org/0000-0002-9151-4650

\section{REFERENCES}

1 Turner JD, Filer A. The role of the synovial fibroblast in rheumatoid arthritis pathogenesis. Curr Opin Rheumatol 2015;27:175-82.

2 Humby F, Lewis M, Ramamoorthi N, et al. Synovial cellular and molecular signatures stratify clinical response to csDMARD therapy and predict radiographic progression in early rheumatoid arthritis patients. Ann Rheum Dis 2019;78:761-72.

3 Lewis MJ, Barnes MR, Blighe K, et al. Molecular portraits of early rheumatoid arthritis identify clinical and treatment response phenotypes. Cell Rep 2019;28:2455-70.
4 Stephenson W, Donlin LT, Butler A, et al. Single-cell RNA-seq of rheumatoid arthritis synovial tissue using low-cost microfluidic instrumentation. Nat Commun 2018;9:791.

5 Mizoguchi F, Slowikowski K, Wei K, et al. Functionally distinct disease-associated fibroblast subsets in rheumatoid arthritis. Nat Commun 2018;9:789.

6 Zhang F, Wei K, Slowikowski K, et al. Defining inflammatory cell states in rheumatoid arthritis joint synovial tissues by integrating single-cell transcriptomics and mass cytometry. Nat Immunol 2019;20:928-42.

7 Alivernini S, MacDonald L, Elmesmari A, et al. Distinct synovial tissue macrophage subsets regulate inflammation and remission in rheumatoid arthritis. Nat Med 2020;26:1295-306.

8 Holweg CTJ, Holweg CTJ, Kummerfeld SK, et al. Synovial phenotypes in rheumatoid arthritis correlate with response to biologic therapeutics. Arthritis Res Ther 2014;16:R90.

9 Timmer TCG, Timmer TCG, Smeets TJ, et al. Fibroblast-like synoviocytes derived from patients with rheumatoid arthritis show the imprint of synovial tissue heterogeneity: evidence of a link between an increased myofibroblast-like phenotype and highinflammation synovitis. Arthritis Rheum 2005;52:430-41.

10 Aletaha D, Neogi T, Silman AJ, et al. 2010 rheumatoid arthritis classification criteria: an American College of Rheumatology/ European League against rheumatism collaborative initiative. Ann Rheum Dis 2010;69:1580-8.

11 Stuart T, Butler A, Hoffman P, et al. Comprehensive integration of single-cell data. Cell 2019;177:1888-902.

12 Welch JD, Kozareva V, Ferreira A, et al. Single-Cell multi-omic integration compares and contrasts features of brain cell identity. Cell 2019;177:1873-87.

13 Korsunsky I, Millard N, Fan J, et al. Fast, sensitive and accurate integration of single-cell data with harmony. Nat Methods 2019;16:1289-96.

14 Tran HTN, Ang KS, Chevrier M, et al. A benchmark of batch-effect correction methods for single-cell RNA sequencing data. Genome Biol 2020;21:12.

15 Trapnell C, Cacchiarelli D, Grimsby J, et al. The dynamics and regulators of cell fate decisions are revealed by pseudotemporal ordering of single cells. Nat Biotechnol 2014;32:381-6.

16 Monocle. Available: http://cole-trapnell-lab.github.io/monoclerelease/

17 Array Express. E-MTAB-6141 - Comprehensive transcriptome analysis of synovium and peripheral blood reveals major disease heterogeneity early in the RA disease process prior to therapeutic intervention. Available: https://www.ebi.ac.uk/arrayexpress/ experiments/E-MTAB-6141/samples/

18 Dobin A, Davis CA, Schlesinger F, et al. STAR: ultrafast universal RNA-seq aligner. Bioinformatics 2013;29:15-21.

19 Liao Y, Smyth GK, Shi W. featureCounts: an efficient general purpose program for assigning sequence reads to genomic features. Bioinformatics 2014;30:923-30.

20 Wang X, Park J, Susztak K, et al. Bulk tissue cell type deconvolution with multi-subject single-cell expression reference. Nat Commun 2019;10:380.

21 GitHub. xuranw / MuSiC. Available: https://github.com/xuranw/ MuSiC

22 Krenn V, Rüther W, Rüther W, et al. 15 years of the histopathological synovitis score, further development and review: a diagnostic score for rheumatology and orthopaedics. Pathol Res Pract 2017;213:874-81.

23 Wei K, Korsunsky I, Marshall JL, et al. Notch signalling drives synovial fibroblast identity and arthritis pathology. Nature 2020;582:259-64.

24 Ospelt C. Synovial fibroblasts in 2017. RMD Open 2017;3:e000471.

25 Galil SMA, EI-Shafey AM, Hagrass HA, et al. Baseline serum level of matrix metalloproteinase-3 as a biomarker of progressive joint damage in rheumatoid arthritis patients. Int $J$ Rheum Dis 2016;19:377-84.

26 Mahmoud RK, El-Ansary AK, El-Eishi HH, et al Matrix metalloproteinases MMP-3 and MMP-1 levels in sera and synovial fluids in patients with rheumatoid arthritis and osteoarthritis. Ital $J$ Biochem $2005 ;: 54: 248-57$.

27 Izquierdo E, Cañete JD, Celis R, et al. Synovial fibroblast hyperplasia in rheumatoid arthritis: clinicopathologic correlations and partial reversal by anti-tumor necrosis factor therapy. Arthritis Rheum 2011;63:2575-83. 